\title{
Existence and Global Attractivity of Pseudo Almost Periodic Solutions for Clifford-Valued Fuzzy Neural Networks with Proportional Delays
}

\author{
Wen Lv and Bing Li *(D) \\ School of Mathematics and Computer Science, Yunnan Minzu University, Kunming 650500, China; \\ lw11292021@163.com \\ * Correspondence: bli123@126.com or libing@ymu.edu.cn
}

\begin{abstract}
In this paper, Clifford-valued fuzzy neural networks with proportional delays, whose leakage term coefficients are also Clifford numbers, are considered. Based on the Banach fixed point theorem and differential inequality technique, we use a direct method to obtain the existence, uniqueness, and global attractivity of pseudo almost periodic solutions for the considered networks. Finally, we provide a numerical example to illustrate the feasibility of our results. Our results are new.
\end{abstract}

Keywords: Clifford-valued neural networks; fuzzy neural networks; proportional delays; pseudo almost periodic solutions; global attractivity

\section{Introduction}

The Clifford-valued neural network is a generalization of a real-valued neural network,

Citation: Lv, W.; Li, B. Existence and Global Attractivity of Pseudo Almost Periodic Solutions for Clifford-Valued Fuzzy Neural Networks with Proportional Delays. Mathematics 2021, 9, 3306. https://doi.org/ $10.3390 /$ math 9243306

Academic Editor: Georgios Tsekouras

Received: 24 November 2021 Accepted: 17 December 2021 Published: 19 December 2021

Publisher's Note: MDPI stays neutral with regard to jurisdictional claims in published maps and institutional affiliations. complex-valued neural network, and quaternion-valued neural network, and has been shown superior to a real-valued neural network [1,2]. Because the multiplication of Clifford algebra does not meet the commutative law, there are few results on the dynamics of Clifford-valued neural networks [3-11]. Many existing results are obtained by decomposing a Clifford-valued system into a real-value system [3-6]. Therefore, it is a meaningful and challenging work to study the dynamics of Clifford-valued systems via a direct method; that is, without decomposing Clifford-valued systems into real-valued systems [7-11].

Since time delay is inevitable in modeling real systems, in recent years, as an important mathematical model, systems with proportional time delays play important roles in many application fields, such as physics, biological systems, neural network systems, and control science $[12,13]$. Therefore, many scholars have studied the dynamics of neural networks with proportional delays. For example, in [14], the existence and global attractiveness of pseudo almost periodic solutions for a class of fuzzy cellular neural networks with multiple proportional delays are obtained by using the fixed point theorem of contractive mapping and differential inequality technique, in [15], the existence, uniqueness, and exponential stability of anti-periodic solutions for recurrent neural networks with multiple delays are established by using the Lyapunov method and inequality technique. In [16], the finite time synchronization problem of a class of fuzzy cellular neural networks with time-varying coefficients and proportional delays is investigated. In [17], a class of competitive neural networks with multiple proportional delays is studied, and a new sufficient condition for the global exponential stability of such a network is obtained.

On the other hand, in the mathematical modeling of practical problems, uncertainty or fuzziness is a factor to be considered. On the basis of traditional cellular neural networks, fuzzy cellular neural networks [18] were first proposed in 1996. They are neural networks that incorporate fuzzy logic into the structure of traditional cellular neural networks. They have important applications in image processing and pattern recognition; therefore, fuzzy neural networks have been widely studied [14-21]. 
In addition, almost periodic oscillation and pseudo almost periodic oscillation are important dynamics of neural networks. Many authors have studied the existence and stability of almost periodic solutions [5,13] and pseudo almost periodic solutions [22-26] of neural networks, including stochastic neural networks [27] and fuzzy neural networks [28,29].

Moreover, recently, the existence and global exponential stability of $(\mu, v)$-pseudo almost periodic solutions to a class of Clifford-valued higher order Hopfield neural networks, with bounded multiple discrete delays whose leakage term coefficients are also Clifford numbers, is studied in [30]. Proportional delays are a class of unbounded delays. Neural networks with proportional delays are a class of neural networks with important theoretical and application values. However, up until now, the results of almost periodic oscillation of neural networks with proportional delays and whose leakage term coefficient $\mathrm{s}$ are also Clifford numbers, have not been reported.

Inspired by the above analysis, the purpose of this paper is to study the existence and attraction of pseudo almost periodic solutions for a class of Clifford-valued fuzzy neural networks with proportional delays and whose leakage term coefficients are also Clifford numbers. To the best of our knowledge, this is the first paper to study the existence and attractivity of pseudo almost periodic solutions of Clifford-valued neural networks with proportional delay and whose leakage coefficients are also Clifford numbers.

The rest of the paper is arranged as follows. In Section 2, we review some concepts, introduce some lemmas, and give the model's description. In Section 3, by using the direct method, we establish the existence of pseudo almost periodic solutions for the network under consideration. In Section 4, we study the global attractivity of pseudo almost periodic solutions. In Section 5, we give a numerical example to illustrate the feasibility of our results. Finally, we draw a brief conclusion in Section 6.

\section{Model Description and Preliminaries}

The real Clifford algebra over $\mathbb{R}^{m}$ is defined as

$$
\mathcal{A}=\left\{\sum_{A \in \Omega} x^{A} e_{A}, x^{A} \in \mathbb{R}\right\}
$$

where $\Omega=\{\varnothing, 1,2, \cdots A, \cdots, 12 \cdots m\}, e_{A}=e_{\hbar_{1}} e_{\hbar_{2}} \cdots e_{\hbar_{k}}=e_{\hbar_{1} \hbar_{2} \cdots \hbar_{k}}$ for $A=\hbar_{1} \hbar_{2} \cdots \hbar_{k}$, $1<\hbar_{1}<\hbar_{2}<\cdots<\hbar_{k}<m$. In addition, $e_{\varnothing}=e_{0}=1$ and $e_{\hbar}, \hbar=1,2, \ldots, m$ are said to be Clifford generators and satisfy $e_{p}^{2}=1, p=1,2, \ldots, s, e_{p}^{2}=-1, p=s+1, s+2, \ldots, m$, $e_{p} e_{q}+e_{q} e_{p}=0, p \neq q$, where $p, q=1,2, \ldots, m$.

For $x=\sum_{A} x^{A} e_{A} \in \mathcal{A}$, we define $\|x\|_{\mathcal{A}}=\max _{A}\left\{\left|x_{A}\right|\right\}$ and for $x=\left(x_{1}, x_{2}, \ldots, x_{n}\right)^{T} \in$ $\mathcal{A}^{n}$, we define $\|x\|_{\mathcal{A}^{n}}=\max _{1 \leq p \leq n}\left\{\left\|x_{p}\right\|_{\mathcal{A}}\right\}$, then $\left.{ }^{A} \mathcal{A},\|\cdot\|_{\mathcal{A}}\right)$ and $\left(\mathcal{A}^{n},\|\cdot\|_{\mathcal{A}^{n}}\right)$ are Banach spaces. For $x=\sum_{A} x^{A} e_{A}$, we define $x^{c}=\sum_{A \neq \varnothing} x^{A} e_{A}$ and $x^{\varnothing}=x-x^{c}$.

According to [19], for $x=\sum x^{A} e_{A}, y=\sum y^{A} e_{A} \in \mathcal{A}$, we define

$$
x \bigvee y=\sum\left(x^{A} \bigvee y^{A}\right) e_{A} \text { and } x \bigwedge y=\sum\left(x^{A} \bigwedge y^{A}\right) e_{A}
$$

where $x^{A} \vee y^{A}=\max \left\{x^{A}, y^{A}\right\}, x^{A} \wedge y^{A}=\min \left\{x^{A}, y^{A}\right\}$.

The model we want to study in this paper is as follows:

$$
\begin{aligned}
\dot{x}_{i}(t)= & -c_{i}(t) x_{i}(t)+\sum_{j=1}^{n} a_{i j}(t) f_{j}\left(x_{j}(t)\right)+\sum_{j=1}^{n} b_{i j}(t) g_{j}\left(x_{j}\left(q_{i j} t\right)\right)+\bigvee_{j=1}^{n} \alpha_{i j}(t) h_{j}\left(x_{j}\left(q_{i j} t\right)\right) \\
& +\bigwedge_{j=1}^{n} \beta_{i j}(t) h_{j}\left(x_{j}\left(q_{i j} t\right)\right)+\bigvee_{j=1}^{n} S_{i j}(t) v_{j}(t)+\bigwedge_{j=1}^{n} T_{i j}(t) v_{j}(t)+I_{i}(t), i=1,2, \ldots, n,
\end{aligned}
$$

where $n$ is the number of neurons in layers, $x_{i}(t) \in \mathcal{A}$ represents the state of the $i$ th unit at time $t, \mathcal{A}$ is a Clifford algebra; $c_{i}(t) \in \mathcal{A}$ is the rate with which the $i$ th unit will reset its 
potential to the resting state in isolation when disconnected from the network and external inputs; $a_{i j}(t), b_{i j}(t) \in \mathcal{A}$ correspond to the first-order and second-order connection weights of the fuzzy neural network; $\alpha_{i j}(t) \in \mathcal{A}$ is the fuzzy feedback MAX template; $\beta_{i j}(t) \in \mathcal{A}$ is the fuzzy feedback MIN template; $S_{i j}(t)$ and $T_{i j}(t) \in \mathcal{A}$ are the fuzzy feed-forward MAX template and the fuzzy feed-forward MIN template; $v_{j}(t) \in \mathcal{A}$ is the input of the $j$ th neuron; $I_{i}(t) \in \mathcal{A}$ stands for the external inputs; $f_{j}(t), g_{j}(t), h_{j}(t) \in \mathcal{A}$ are the activation functions; $0<q_{i j}<1$ is the proportional coefficient of proportional delay.

For convenience, we will adopt the following notations:

$$
\begin{array}{ccc}
\bar{c}_{i}^{\varnothing}=\inf _{t \in \mathbb{R}}\left\{\left\|c_{i}^{\varnothing}(t)\right\|_{\mathcal{A}}\right\}, & \bar{c}_{i}^{c}=\sup _{t \in \mathbb{R}}\left\{\left\|c_{i}^{c}(t)\right\|_{\mathcal{A}}\right\}, & a_{i j}^{+}=\sup _{t \in \mathbb{R}}\left\{\left\|a_{i j}(t)\right\|_{\mathcal{A}}\right\}, \\
b_{i j}^{+}=\sup _{t \in \mathbb{R}}\left\{\left\|b_{i j}(t)\right\|_{\mathcal{A}}\right\}, & \alpha_{i j}^{+}=\sup _{t \in \mathbb{R}}\left\{\left\|\alpha_{i j}(t)\right\|_{\mathcal{A}}\right\}, & \beta_{i j}^{+}=\sup _{t \in \mathbb{R}}\left\{\left\|\beta_{i j}(t)\right\|_{\mathcal{A}}\right\},
\end{array}
$$

The initial values of system (1) are given by

$$
x_{i}(s)=\varphi_{i}(s), \quad s \in\left[q t_{0}, t_{0}\right],
$$

where $\varphi_{i} \in C\left(\left[q t_{0}, t_{0}\right], \mathbb{R}\right), q=\min _{1 \leq j \leq n}\left\{q_{i j}\right\}$.

Let $B C\left(\mathbb{R}, \mathcal{A}^{n}\right)$ be the collection of all bounded and continuous functions from $\mathbb{R}$ to $\mathcal{A}^{n}$. The space $\left(B C\left(\mathbb{R}, \mathcal{A}^{n}\right),\|\cdot\|_{0}\right)$ is a Banach space when it is endowed with the norm:

$$
\|f\|_{0}=\sup _{t \in \mathbb{R}}\|f(t)\|_{\mathcal{A}^{n}}
$$

where $f \in B C\left(\mathbb{R}, \mathcal{A}^{n}\right)$.

Definition 1 ([20]). A function $f \in B C\left(\mathbb{R}, \mathcal{A}^{n}\right)$ is called to an almost periodic function, if for every $\varepsilon>0$, there exists $l=l(\varepsilon)>0$ such that for each interval with length $l(\varepsilon)$, one can find a number $\tau \in[a, a+l(\varepsilon)]$ satisfying

$$
\|f(t+\tau)-f(t)\|_{\mathcal{A}^{n}}<\varepsilon, \forall t \in \mathbb{R} .
$$

All of these functions will be denoted by $A P\left(\mathbb{R}, \mathcal{A}^{n}\right)$.

Definition 2 ([20]). A function $f \in B C\left(\mathbb{R}, \mathcal{A}^{n}\right)$ is said to be pseudo almost periodic, if it can be written as $f=f_{1}+f_{2}$, where $f_{1} \in A P\left(\mathbb{R}, \mathcal{A}^{n}\right), f_{2} \in P A P_{0}\left(\mathbb{R}, \mathcal{A}^{n}\right)$ and

$$
\operatorname{PAP}_{0}\left(\mathbb{R}, \mathcal{A}^{n}\right)=\left\{f \in B C\left(\mathbb{R}, \mathcal{A}^{n}\right) \mid \lim _{T \rightarrow+\infty} \frac{1}{2 T} \int_{-T}^{T}\|f(t)\|_{\mathcal{A}^{n}} d t=0\right\} .
$$

Lemma 1 ([20]). If $f, g \in P A P\left(\mathbb{R}, \mathcal{A}^{n}\right)$, then $f+g, f g \in P A P\left(\mathbb{R}, \mathcal{A}^{n}\right)$.

Lemma 2 ([20]). Let $f \in C\left(\mathcal{A}, \mathcal{A}^{n}\right)$ satisfy the Lipschitz condition and $g \in P A P\left(\mathbb{R}, \mathcal{A}^{n}\right)$, then $f(g(\cdot)) \in \operatorname{PAP}\left(\mathbb{R}, \mathcal{A}^{n}\right)$.

Lemma 3 ( [21]). If $0<q<1, t \in \mathbb{R}, x \in P A P\left(\mathbb{R}, \mathcal{A}^{n}\right)$, then $x(q t) \in P A P\left(\mathbb{R}, \mathcal{A}^{n}\right)$.

Using the same proof method as Lemma 2 in [14], we can prove

Lemma 4. Assume that $x, y \in P A P(\mathbb{R}, \mathcal{A})$, then we have $x \bigvee y, x \wedge y \in P A P(\mathbb{R}, \mathcal{A})$.

Lemma 5 ([20]). The space $\left(\operatorname{PAP}\left(\mathbb{R}, \mathcal{A}^{n}\right),\|\cdot\|_{0}\right)$ is a Banach space.

Similar to the proof of Corollary 1 in [18], one can easily show that 
Lemma 6. If $\alpha_{i j}, \beta_{i j} \in C(\mathbb{R}, \mathcal{A}), f \in C(\mathbb{R}, \mathcal{A}), x, y \in \mathcal{A}$. Then we have

$$
\begin{aligned}
& \left\|\bigwedge_{j=1}^{n} \alpha_{i j}(t) f_{j}(x)-\bigwedge_{j=1}^{n} \alpha_{i j}(t) f_{j}(y)\right\|_{\mathcal{A}} \leq \sum_{j=1}^{n}\left\|\alpha_{i j}(t)\right\|_{\mathcal{A}}\left\|f_{j}(x)-f_{j}(y)\right\|_{\mathcal{A}}, \\
& \left\|\bigvee_{j=1}^{n} \beta_{i j}(t) f_{j}(x)-\bigvee_{j=1}^{n} \beta_{i j}(t) f_{j}(y)\right\|_{\mathcal{A}} \leq \sum_{j=1}^{n}\left\|\beta_{i j}(t)\right\|_{\mathcal{A}}\left\|f_{j}(x)-f_{j}(y)\right\|_{\mathcal{A}} .
\end{aligned}
$$

Lemma 7 ([20]). Let $G(t)=\int_{-\infty}^{t} e^{-\int_{s}^{t} a(u) d u} F(s) d s$, where $a \in A P\left(\mathbb{R}, \mathbb{R}^{+}\right)$with $\inf _{t \in \mathbb{R}} a(t)>0$ and $F \in \operatorname{PAP}\left(\mathbb{R}, \mathcal{A}^{n}\right)$, then $G \in \operatorname{PAP}\left(\mathbb{R}, \mathcal{A}^{n}\right)$.

The assumptions used in this paper are as follows:

$\left(H_{1}\right)$ For $i, j=1,2, \ldots n, c_{i}^{\varnothing} \in A P\left(\mathbb{R}, \mathbb{R}^{+}\right)$with $\bar{c}_{i}^{\varnothing}>0, c_{i}^{c}, a_{i j}, b_{i j}, \alpha_{i j}, \beta_{i j} \in P A P(\mathbb{R}, \mathcal{A})$, $S_{i j}, T_{i j}, I_{i} \in \operatorname{PAP}(\mathbb{R}, \mathcal{A})$.

$\left(H_{2}\right)$ For $j=1,2, \cdots n$, functions $f_{j}, g_{j}, h_{j} \in C(\mathcal{A}, \mathcal{A})$ satisfy there exist constants $K_{j}, L_{j}, M_{j}, N_{j}$, such that for any $s, t \in \mathcal{A}$,

$$
\begin{aligned}
& \left\|f_{j}(s)-f_{j}(t)\right\|_{\mathcal{A}} \leq K_{j}\|s-t\|_{\mathcal{A}}, \quad\left\|g_{j}(s)-g_{j}(t)\right\|_{\mathcal{A}} \leq L_{j}\|s-t\|_{\mathcal{A}}, \\
& \left\|h_{j}(s)-h_{j}(t)\right\|_{\mathcal{A}} \leq M_{j}\|s-t\|_{\mathcal{A}}, \quad\left\|I_{j}(s)-I_{j}(t)\right\|_{\mathcal{A}} \leq N_{j}\|s-t\|_{\mathcal{A}},
\end{aligned}
$$

and $f_{j}(0)=g_{j}(0)=h_{j}(0)=0$.

$\left(H_{3}\right)$ The constant

$$
\delta:=\max _{1 \leq i \leq n}\left\{\frac{1}{\bar{c}_{i}^{\varnothing}}\left(\bar{c}_{i}^{c}+\sum_{j=1}^{n} a_{i j}^{+} K_{j}+\sum_{j=1}^{n} b_{i j}^{+} L_{j}+\sum_{j=1}^{n} \alpha_{i j}^{+} M_{j}+\sum_{j=1}^{n} \beta_{i j}^{+} M_{j}\right)\right\}<1 .
$$

\section{Pseudo Almost Periodic Solutions}

In this section, by the contracting mapping principle, we will study the existence of pseudo almost periodic solutions of (1).

For $i, j=1,2, \ldots, n$, we define

$$
\begin{aligned}
F_{i}(t, x)= & -c_{i}^{c}(t) x_{i}(t)+\sum_{j=1}^{n} a_{i j}(t) f_{j}\left(x_{j}(t)\right)+\sum_{j=1}^{n} b_{i j}(t) g_{j}\left(x_{j}\left(q_{i j} t\right)\right)+\bigvee_{j=1}^{n} \alpha_{i j}(t) h_{j}\left(x_{j}\left(q_{i j} t\right)\right) \\
& +\bigwedge_{j=1}^{n} \beta_{i j}(t) h_{j}\left(x_{j}\left(q_{i j} t\right)\right)+I_{i}(t), i=1,2, \ldots, n .
\end{aligned}
$$

Obviously, if $x=\left(x_{1}, x_{2}, \cdots x_{n}\right)^{T}$ is a solution of the integral equation:

$$
x_{i}(t)=\int_{-\infty}^{t} e^{-\int_{s}^{t} c_{i}^{\varnothing}(u) d u}\left[F_{i}(s, x)+\bigvee_{j=1}^{n} S_{i j}(s) v_{j}(s)+\bigwedge_{j=1}^{n} T_{i j}(s) v_{j}(s)+I_{i}(s)\right] d s, i=1,2, \ldots, n,
$$

then $x$ is a solution of system (1).

Let $B=\left(P A P\left(\mathbb{R}, \mathcal{A}^{n}\right),\|\cdot\|_{0}\right)$ and

$$
\begin{aligned}
\tilde{\varphi}(t)= & \left(\int_{-\infty}^{t} e^{-\int_{s}^{t} c_{1}^{\varnothing}(u) d u}\left(\bigvee_{j=1}^{n} S_{1 j}(s) v_{j}(s)+\bigwedge_{j=1}^{n} T_{1 j}(s) v_{j}(s)+I_{1}(s)\right) d s, \cdots,\right. \\
& \left.\int_{-\infty}^{t} e^{-\int_{s}^{t} c_{n}^{\varnothing}(u) d u}\left(\bigvee_{j=1}^{n} S_{n j}(s) v_{j}(s)+\bigwedge_{j=1}^{n} T_{n j}(s) v_{j}(s)+I_{n}(s)\right) d s\right)^{T} .
\end{aligned}
$$


Define

$$
B_{\delta}=\left\{\varphi \in B \mid\|\varphi-\tilde{\varphi}\|_{0} \leq \frac{\delta N}{1-\delta}\right\}
$$

where $\delta$ is mentioned in $\left(H_{3}\right)$ and $N$ is a positive constant satisfying $N \geq\|\tilde{\varphi}\|_{0}$, then we have

$$
\|\varphi\|_{0} \leq\|\varphi-\tilde{\varphi}\|_{0}+\|\tilde{\varphi}\|_{0} \leq \frac{\delta N}{1-\delta}+N=\frac{N}{1-\delta}
$$

Theorem 1. If assumptions $\left(H_{1}\right)-\left(H_{3}\right)$ hold, then system (1) has a unique pseudo almost periodic solution in $B_{\delta}=\left\{\varphi \in B \mid\|\varphi-\tilde{\varphi}\|_{0} \leq \frac{\delta N}{1-\delta}\right\}$.

Proof. Consider the mapping $T: B \rightarrow B C\left(\mathbb{R}, \mathcal{A}^{n}\right)$ defined by setting $T \varphi=\left(T_{1} \varphi, T_{2} \varphi, \cdots\right.$, $\left.T_{n} \varphi\right)^{T}$, where

$$
\left(T_{i} \varphi\right)(t)=\int_{-\infty}^{t} e^{-\int_{s}^{t} c_{i}^{\varnothing}(u) d u}\left[F_{i}(s, x)+\bigvee_{j=1}^{n} S_{i j}(s) v_{j}(s)+\bigwedge_{j=1}^{n} T_{i j}(s) v_{j}(s)+I_{i}(s)\right] d s .
$$

By Lemmas 1-4, we obtain $F_{i} \in \operatorname{PAP}(\mathbb{R}, \mathcal{A})$. Further, by Lemma 7 , we have $T \varphi \in$ $\operatorname{PAP}(\mathbb{R}, \mathcal{A})$.

We will divide the rest of the proof into two steps.

Step 1 , we will verify $T: B_{\delta} \rightarrow B_{\delta}$ is a self-mapping. For any $\varphi \in B_{\delta}$, we have

$$
\begin{aligned}
& \|T \varphi-\tilde{\varphi}\|_{0} \leq \sup _{t \in \mathbb{R}}\left\{\operatorname { m a x } _ { 1 \leq i \leq n } \left\{\int _ { - \infty } ^ { t } e ^ { - \int _ { s } ^ { t } c _ { i } ^ { \varnothing } ( u ) d u } \left(\left\|c_{i}^{\mathcal{c}}(s) \varphi_{i}(s)\right\|_{\mathcal{A}}+\sum_{j=1}^{n}\left\|a_{i j}(s) f_{j}\left(\varphi_{j}(s)\right)\right\|_{\mathcal{A}}\right.\right.\right. \\
& +\sum_{j=1}^{n}\left\|b_{i j}(s) g_{j}\left(\varphi_{j}\left(q_{i j} s\right)\right)\right\|_{\mathcal{A}}+\sum_{j=1}^{n}\left\|\alpha_{i j}(s) h_{j}\left(\varphi_{j}\left(q_{i j} s\right)\right)\right\|_{\mathcal{A}} \\
& \left.\left.\left.+\sum_{j=1}^{n}\left\|\beta_{i j}(s) h_{j}\left(\varphi_{j}\left(q_{i j} s\right)\right)\right\|_{\mathcal{A}}\right) d s\right\}\right\} \\
& \leq \sup _{t \in \mathbb{R}}\left\{\operatorname { m a x } _ { 1 \leq i \leq n } \left\{\int _ { - \infty } ^ { t } e ^ { - \int _ { s } ^ { t } c _ { i } ^ { \varnothing } ( u ) d u } \left(\bar{c}_{i}^{c}\|\varphi\|_{0}+\sum_{j=1}^{n} a_{i j}^{+} K_{j}\|\varphi\|_{0}+\sum_{j=1}^{n} b_{i j}^{+} L_{j}\|\varphi\|_{0}\right.\right.\right. \\
& \left.\left.\left.+\sum_{j=1}^{n} \alpha_{i j}^{+} M_{j}\|\varphi\|_{0}+\sum_{j=1}^{n} \beta_{i j}^{+} M_{j}\|\varphi\|_{0}\right) d s\right\}\right\} \\
& \leq \max _{1 \leq i \leq n}\left\{\frac{1}{\bar{c}_{i}^{\varnothing}}\left(\bar{c}_{i}^{c}+\sum_{j=1}^{n}\left[a_{i j}^{+} K_{j}+b_{i j}^{+} L_{j}+\alpha_{i j}^{+} M_{j}+\beta_{i j}^{+} M_{j}\right]\right)\right\}\|\varphi\|_{0} \\
& \leq \frac{\delta N}{1-\delta} \text {. }
\end{aligned}
$$

Hence, $\varphi\left(B_{\delta}\right) \subset B_{\delta}$. 
Step 2, we will show $T$ is a contractive mapping. For any $\varphi, \psi \in B_{\delta}$, we find

$$
\begin{aligned}
& \|T \varphi-T \psi\|_{0} \leq \sup _{t \in \mathbb{R}}\left\{\operatorname { m a x } _ { 1 \leq i \leq n } \left\{\int _ { - \infty } ^ { t } e ^ { - \int _ { s } ^ { t } c _ { i } ^ { \varnothing } ( u ) d u } \left(\left\|c_{i}^{c}(s)\left(\varphi_{i}(s)-\psi_{i}(s)\right)\right\|_{\mathcal{A}}\right.\right.\right. \\
& +\sum_{j=1}^{n}\left\|a_{i j}(s)\left(f_{j}\left(\varphi_{j}(s)\right)-f_{j}\left(\psi_{j}(s)\right)\right)\right\|_{\mathcal{A}} \\
& +\sum_{j=1}^{n}\left\|b_{i j}(s)\left(g_{j}\left(\varphi_{j}\left(q_{i j} s\right)\right)-g_{j}\left(\psi_{j}\left(q_{i j} s\right)\right)\right)\right\|_{\mathcal{A}} \\
& +\sum_{j=1}^{n}\left\|\alpha_{i j}(s)\left(h_{j}\left(\varphi_{j}\left(q_{i j} s\right)\right)-h_{j}\left(\psi_{j}\left(q_{i j} s\right)\right)\right)\right\|_{\mathcal{A}} \\
& \left.\left.\left.+\sum_{j=1}^{n}\left\|\beta_{i j}(s)\left(h_{j}\left(\varphi_{j}\left(q_{i j} s\right)\right)-h_{j}\left(\psi_{j}\left(q_{i j} s\right)\right)\right)\right\|_{\mathcal{A}}\right) d s\right\}\right\} \\
& \leq \sup _{t \in \mathbb{R}}\left\{\operatorname { m a x } _ { 1 \leq i \leq n } \left\{\int _ { - \infty } ^ { t } e ^ { - \int _ { s } ^ { t } c _ { i } ^ { \varnothing } ( u ) d u } \left(\bar{c}_{i}^{c}\|\varphi-\psi\|_{0}\right.\right.\right. \\
& +\sum_{j=1}^{n} a_{i j}^{+} K_{j}\|\varphi-\psi\|_{0}+\sum_{j=1}^{n} b_{i j}^{+} L_{j}\|\varphi-\psi\|_{0} \\
& \left.\left.\left.+\sum_{j=1}^{n} \alpha_{i j}^{+} M_{j}\|\varphi-\psi\|_{0}+\sum_{j=1}^{n} \beta_{i j}^{+} M_{j}\|\varphi-\psi\|_{0}\right) d s\right\}\right\} \\
& \leq \max _{1 \leq i \leq n}\left\{\frac { 1 } { \overline { c } _ { i } ^ { \varnothing } } \left(\bar{c}_{i}^{c}+\sum_{j=1}^{n} a_{i j}^{+} K_{j}+\sum_{j=1}^{n} b_{i j}^{+} L_{j}\right.\right. \\
& \left.\left.+\sum_{j=1}^{n} \alpha_{i j}^{+} M_{j}+\sum_{j=1}^{n} \beta_{i j}^{+} M_{j}\right)\right\}\|\varphi-\psi\|_{0} \\
& \leq \delta\|\varphi-\psi\|_{0} .
\end{aligned}
$$

Noticing that $\delta<1, T$ is a contracting mapping. Therefore, system (1) possesses a unique one pseudo almost periodic solution in $B_{\delta}$. This completes the proof.

\section{Global Attractivity}

Our result on the global attractivity of pseudo almost periodic solutions of system (1) is as follows:

Theorem 2. If conditions $\left(H_{1}\right)-\left(H_{3}\right)$ hold, then system (1) has a unique pseudo almost periodic solution $x$ satisfying that there exist constants $\lambda>0$ and $M>1$, such that

$$
\|x(t)-y(t)\|_{\mathcal{A}^{n}} \leq M\left(\|\varphi-\psi\|_{\xi}+\varepsilon\right) e^{-\lambda \ln \frac{1+t}{1+t_{0}}}, t>t_{0},
$$

where $y$ is an arbitrary solution of system (1) with initial value $\varphi, \psi$ is the initial value of $x$ and $\|\varphi-\psi\|_{\xi}=\sup _{s \in\left[q t_{0}, t_{0}\right]}\|\varphi(s)-\psi(s)\|_{\mathcal{A}^{n}}$.

Proof. If $x(t)$ is the pseudo almost periodic solution of system (1) with initial value $\varphi$ and $y$ is an arbitrary solution of system (1) with the initial value $\psi$. Let $z(t)=x(t)-y(t)$. Then, for $i=1,2, \ldots, n$, one gets 


$$
\begin{aligned}
z_{i}^{\prime}(t)= & -c_{i}(t) z_{i}(t)+\sum_{j=1}^{n} a_{i j}(t)\left(f_{j}\left(x_{j}(t)\right)-f_{j}\left(y_{j}(t)\right)\right)+\sum_{j=1}^{n} b_{i j}(t)\left(g_{j}\left(x_{j}\left(q_{i j} t\right)\right)-g_{j}\left(x_{j}\left(q_{i j} t\right)\right)\right) \\
& +\bigvee_{j=1}^{n} \alpha_{i j}(t)\left(h_{j}\left(x_{j}\left(q_{i j} t\right)\right)-h_{j}\left(y_{j}\left(q_{i j} t\right)\right)\right)+\bigwedge_{j=1}^{n} \beta_{i j}(t)\left(h_{j}\left(x_{j}\left(q_{i j} t\right)\right)-h_{j}\left(y_{j}\left(q_{i j} t\right)\right)\right) .
\end{aligned}
$$

From $\left(H_{3}\right)$, for $i=1,2, \ldots, n$, we have

$$
\begin{gathered}
\sup _{t \geq 0}\left\{-\bar{c}_{i}^{\varnothing}+\left(\left\|c_{i}^{c}(t)\right\|_{\mathcal{A}}+\sum_{j=1}^{n}\left\|a_{i j}(t)\right\|_{\mathcal{A}} K_{j}+\sum_{j=1}^{n}\left\|b_{i j}(t)\right\|_{\mathcal{A}} L_{j}\right.\right. \\
\left.\left.\quad+\sum_{j=1}^{n}\left\|\alpha_{i j}(t)\right\|_{\mathcal{A}} M_{j}+\sum_{j=1}^{n}\left\|\beta_{i j}(t)\right\|_{\mathcal{A}} M_{j}\right)\right\}<0 .
\end{gathered}
$$

Let $K_{i}$ be defined by

$$
\begin{aligned}
K_{i}(\theta)= & \sup _{t \in \mathbb{R}}\left\{\theta-\bar{c}_{i}^{\varnothing}+\left(\left\|c_{i}^{c}(t)\right\|_{\mathcal{A}}+\sum_{j=1}^{n}\left\|a_{i j}(t)\right\|_{\mathcal{A}} K_{j}+\sum_{j=1}^{n}\left\|b_{i j}(t)\right\|_{\mathcal{A}} L_{j} e^{\theta \ln \frac{1}{q_{i j}}}\right.\right. \\
& \left.\left.+\sum_{j=1}^{n}\left\|\alpha_{i j}(t)\right\|_{\mathcal{A}} M_{j} e^{\theta \ln \frac{1}{q_{i j}}}+\sum_{j=1}^{n}\left\|\beta_{i j}(t)\right\|_{\mathcal{A}} M_{j} e^{\theta \ln \frac{1}{q_{i j}}}\right)\right\}, i=1,2, \ldots, n,
\end{aligned}
$$

where $\theta \in\left[0, \min _{1 \leq i \leq n} \bar{c}_{i}^{\varnothing}\right]$. Then, from (3), we have

$$
\begin{aligned}
K_{i}(0)= & \sup _{t \in \mathbb{R}}\left\{-\bar{c}_{i}^{\varnothing}+\left(\left\|c_{i}^{c}(t)\right\|_{\mathcal{A}}+\sum_{j=1}^{n}\left\|a_{i j}(t)\right\|_{\mathcal{A}} K_{j}+\sum_{j=1}^{n}\left\|b_{i j}(t)\right\|_{\mathcal{A}} L_{j}\right.\right. \\
& \left.\left.+\sum_{j=1}^{n}\left\|\alpha_{i j}(t)\right\|_{\mathcal{A}} M_{j}+\sum_{j=1}^{n}\left\|\beta_{i j}(t)\right\|_{\mathcal{A}} M_{j}\right)\right\}<0, i=1,2, \ldots, n .
\end{aligned}
$$

Since $K_{i}(0)=0, K_{i}(\theta) \rightarrow \infty$ as $\theta \rightarrow \infty$ and the continuity of $K_{i}(\theta)$, we can take a constant $\lambda \in\left(0, \min _{1 \leq i \leq n}\left\{\bar{c}_{i}^{\varnothing}\right\}\right)$, such that $K_{i}(\lambda)<0$ and

$$
\begin{aligned}
\sup _{t \in \mathbb{R}}\{ & \frac{\lambda}{1+t}-\bar{c}_{i}^{\varnothing}+\left(\left\|c_{i}^{c}(t)\right\|_{\mathcal{A}}+\sum_{j=1}^{n}\left\|a_{i j}(t)\right\|_{\mathcal{A}} K_{j}+\sum_{j=1}^{n}\left\|b_{i j}(t)\right\|_{\mathcal{A}} L_{j} e^{\lambda \ln \frac{1}{q_{i j}}}\right. \\
& \left.\left.+\sum_{j=1}^{n}\left\|\alpha_{i j}(t)\right\|_{\mathcal{A}} M_{j} e^{\lambda \ln \frac{1}{q_{i j}}}+\sum_{j=1}^{n}\left\|\beta_{i j}(t)\right\|_{\mathcal{A}} M_{j} e^{\lambda \ln \frac{1}{q_{i j}}}\right)\right\} \\
< & \sup _{t \in \mathbb{R}}\left\{\lambda-\bar{c}_{i}^{\varnothing}+\left(\left\|c_{i}^{c}(t)\right\|_{\mathcal{A}}+\sum_{j=1}^{n}\left\|a_{i j}(t)\right\|_{\mathcal{A}} K_{j}+\sum_{j=1}^{n}\left\|b_{i j}(t)\right\|_{\mathcal{A}} L_{j} e^{\lambda \ln \frac{1}{q_{i j}}}\right.\right. \\
& \left.\left.+\sum_{j=1}^{n}\left\|\alpha_{i j}(t)\right\|_{\mathcal{A}} M_{j} e^{\lambda \ln \frac{1}{q_{i j}}}+\sum_{j=1}^{n}\left\|\beta_{i j}(t)\right\|_{\mathcal{A}} M_{j} e^{\lambda \ln \frac{1}{q_{i j}}}\right)\right\}<0, i=1,2, \ldots, n .
\end{aligned}
$$

Noting that

$$
\ln \frac{1+t}{1+q_{i j} t} \leq \ln \frac{1}{q_{i j}}, t \geq 0, i, j=1,2, \ldots, n,
$$

for any $\varepsilon>0, M>1$, we have that

$$
\|z(t)\|_{\mathcal{A}^{n}} \leq M\left(\|\varphi-\psi\|_{\xi}+\varepsilon\right) e^{-\lambda \ln \frac{1+t}{1+t_{0}}}, t \in\left[q t_{0}, t_{0}\right] .
$$


We claim that

$$
\|z(t)\|_{\mathcal{A}^{n}} \leq M\left(\|\varphi-\psi\|_{\xi}+\varepsilon\right) e^{-\lambda \ln \frac{1+t}{1+t_{0}}}, t>t_{0}
$$

To prove (4) holds, we will prove for every $d>1$, the following inequality holds.

$$
\|z(t)\|_{\mathcal{A}^{n}} \leq d M\left(\|\varphi-\psi\|_{\xi}+\varepsilon\right) e^{-\lambda \ln \frac{1+t}{1+t_{0}}}, t \in\left[t_{0},+\infty\right) .
$$

If (5) does not hold, then there must be some $\theta>1$, such that

$$
\left\{\begin{array}{l}
\|z(\theta)\|_{\mathcal{A}^{n}}=d M\left(\|\varphi-\psi\|_{\xi}+\varepsilon\right) e^{-\lambda \ln \frac{1+\theta}{1+t_{0}}} \\
\|z(t)\|_{\mathcal{A}^{n}}<d M\left(\|\varphi-\psi\|_{\xi}+\varepsilon\right) e^{-\lambda \ln \frac{1+t}{1+t_{0}}}, t \in\left[q t_{0}, \theta\right),
\end{array}\right.
$$

which combined with (2), for $i=1,2, \ldots, n$, we have

$$
\begin{aligned}
& \left\|z_{i}(\theta)\right\|_{\mathcal{A}}=\| z_{i}\left(t_{0}\right) e^{-\int_{t_{0}}^{\theta} c_{i}^{\varnothing}(u) d u}+\int_{t_{0}}^{\theta} e^{-\int_{s}^{\theta} c_{i}^{\varnothing}(u) d u}\left(c_{i}^{c}(s) z_{i}(s)\right. \\
& +\sum_{j=1}^{n} a_{i j}(s)\left(f_{j}\left(x_{j}(s)\right)-f_{j}\left(y_{j}(s)\right)\right)+\sum_{j=1}^{n} b_{i j}(s)\left(g_{j}\left(x_{j}\left(q_{i j} s\right)\right)-g_{j}\left(y_{j}\left(q_{i j} s\right)\right)\right) \\
& +\sum_{j=1}^{n} \beta_{i j}(s)\left(h_{j}\left(x_{j}\left(q_{i j} s\right)\right)-h_{j}\left(y_{j}\left(q_{i j} s\right)\right)\right) \\
& \left.+\sum_{j=1}^{n} \alpha_{i j}(s)\left(h_{j}\left(x_{j}\left(q_{i j} s\right)\right)-h_{j}\left(y_{j}\left(q_{i j} s\right)\right)\right) d s \|_{\mathcal{A}}\right) \\
& \leq\left\|z_{i}\left(t_{0}\right)\right\|_{\mathcal{A}} e^{-\int_{t_{0}}^{\theta} \bar{c}_{i}^{\varnothing} d u}+\int_{t_{0}}^{\theta} e^{-\int_{s}^{\theta} \bar{c}_{i}^{\varnothing} d u}\left(\left\|c_{i}^{\mathcal{C}}(t)\right\|_{\mathcal{A}}\left\|z_{i}(s)\right\|_{\mathcal{A}}\right. \\
& +\sum_{j=1}^{n}\left\|a_{i j}(s)\right\|_{\mathcal{A}} K_{j}\left\|z_{j}(s)\right\|_{\mathcal{A}}+\sum_{j=1}^{n}\left\|b_{i j}(s)\right\|_{\mathcal{A}} L_{j}\left\|z_{j}\left(q_{i j} s\right)\right\|_{\mathcal{A}} \\
& \left.+\sum_{j=1}^{n}\left\|\alpha_{i j}(s)\right\|_{\mathcal{A}} M_{j}\left\|z_{j}\left(q_{i j} s\right)\right\|_{\mathcal{A}}+\sum_{j=1}^{n}\left\|\beta_{i j}(s)\right\|_{\mathcal{A}} M_{j}\left\|z_{j}\left(q_{i j} s\right)\right\|_{\mathcal{A}}\right) d s \\
& \leq M\left(\|\varphi-\psi\|_{\xi}+\varepsilon\right) e^{-\int_{t_{0}}^{\theta} \bar{c}_{i}^{\varnothing} d u}+d M\left(\|\varphi-\psi\|_{\xi}+\varepsilon\right) \int_{t_{0}}^{\theta} e^{-\int_{s}^{\theta} \bar{c}_{i}^{\varnothing} d u}\left(\left\|c_{i}^{c}(s)\right\|_{\mathcal{A}} e^{-\lambda \ln \frac{1+s}{1+t_{0}}}\right. \\
& +\sum_{j=1}^{n}\left\|a_{i j}(s)\right\|_{\mathcal{A}} K_{j} e^{-\lambda \ln \frac{1+s}{1+t_{0}}}+\sum_{j=1}^{n}\left\|b_{i j}(s)\right\|_{\mathcal{A}} L_{j} e^{-\lambda \ln \frac{1+q_{i j} s}{1+t_{0}}} \\
& \left.+\sum_{j=1}^{n}\left\|\alpha_{i j}(s)\right\|_{\mathcal{A}} M_{j} e^{-\lambda \ln \frac{1+q_{i j} s}{1+t_{0}}}+\sum_{j=1}^{n}\left\|\beta_{i j}(s)\right\|_{\mathcal{A}} M_{j} e^{-\lambda \ln \frac{1+q_{i j} s}{1+t_{0}}}\right) d s \\
& =d M\left(\|\varphi-\psi\|_{\xi}+\varepsilon\right) e^{-\lambda \ln \frac{1+\theta}{1+t_{0}}}\left(\frac{1}{d} e^{-\int_{t_{0}}^{\theta}\left(\bar{c}_{i}^{\varnothing}-\frac{\lambda}{1+u}\right) d u}\right. \\
& +\int_{t_{0}}^{\theta} e^{-\int_{s}^{\theta}\left(\bar{c}_{i}^{\varnothing}-\frac{\lambda}{1+u}\right) d u}\left(\left\|c_{i}^{c}(s)\right\|_{\mathcal{A}}+\sum_{j=1}^{n}\left\|a_{i j}(s)\right\|_{\mathcal{A}} K_{j}+\sum_{j=1}^{n}\left\|b_{i j}(s)\right\|_{\mathcal{A}} L_{j} e^{\lambda \ln \frac{1+s}{1+q_{i j}}}\right. \\
& \left.\left.+\sum_{j=1}^{n}\left\|\alpha_{i j}(s)\right\|_{\mathcal{A}} M_{j} e^{\lambda \ln \frac{1+s}{1+q_{i j} s}}+\sum_{j=1}^{n}\left\|\beta_{i j}(s)\right\|_{\mathcal{A}} M_{j} e^{\lambda \ln \frac{1+s}{1+q_{i j} s}}\right) d s\right)
\end{aligned}
$$




$$
\begin{aligned}
& \leq d M\left(\|\varphi-\psi\|_{\xi}+\varepsilon\right) e^{-\lambda \ln \frac{1+\theta}{1+t_{0}}}\left(\frac{1}{d} e^{-\int_{t_{0}}^{\theta}\left(\bar{c}_{i}^{\varnothing}-\frac{\lambda}{1+u}\right) d u}\right. \\
&+\int_{t_{0}}^{\theta} e^{-\int_{s}^{\theta}\left(\bar{c}_{i}^{\varnothing}-\frac{\lambda}{1+u}\right) d u}\left(\left\|c_{i}^{c}(s)\right\|_{\mathcal{A}}+\sum_{j=1}^{n}\left\|a_{i j}(s)\right\|_{\mathcal{A}} K_{j}+\sum_{j=1}^{n}\left\|b_{i j}(s)\right\|_{\mathcal{A}} L_{j} e^{\lambda \ln \frac{1}{q_{i j}}}\right. \\
&\left.\left.+\sum_{j=1}^{n}\left\|\alpha_{i j}(s)\right\|_{\mathcal{A}} M_{j} e^{\lambda \ln \frac{1}{q_{i j}}}+\sum_{j=1}^{n}\left\|\beta_{i j}(s)\right\|_{\mathcal{A}} M_{j} e^{\lambda \ln \frac{1}{q_{i j}}}\right) d s\right) \\
& \leq d M\left(\|\varphi-\psi\|_{\xi}+\varepsilon\right) e^{-\lambda \ln \frac{1+\theta}{1+t_{0}}}\left(\frac{1}{d} e^{-\int_{t_{0}}^{\theta}\left(\bar{c}_{i}^{\varnothing}-\frac{\lambda}{1+u}\right) d u}\right. \\
&\left.+\int_{t_{0}}^{\theta} e^{-\int_{s}^{\theta}\left(\bar{c}_{i}^{\varnothing}-\frac{\lambda}{1+u}\right) d u}\left(\bar{c}_{i}^{\varnothing}-\frac{\lambda}{1+s}\right) d s\right) \\
& \leq d M\left(\|\varphi-\psi\|_{\xi}+\varepsilon\right) e^{-\lambda \ln \frac{1+\theta}{1+t_{0}}}\left(1-\left(1-\frac{1}{d}\right) e^{-\int_{t_{0}}^{\theta}\left(\bar{c}_{i}^{\varnothing}-\frac{\lambda}{1+u}\right) d u}\right) \\
&<d M\left(\|\varphi-\psi\|_{\xi}+\varepsilon\right) e^{-\lambda \ln \frac{1+\theta}{1+t_{0}}}
\end{aligned}
$$

this yields that,

$$
\|z(\theta)\|_{\mathcal{A}^{n}}<d M\left(\|\varphi-\psi\|_{\mathcal{\zeta}}+\varepsilon\right) e^{-\lambda \ln \frac{1+\theta}{1+t_{0}}},
$$

which contradicts (6). Hence, (5) holds. Letting $d \rightarrow 1$, we derive that (4) holds. The proof is complete.

\section{An Example}

Our numerical example is as follows.

Example 1. In system (1), let $n=m=2, s=0$ and for $i, j=1,2$, take

$$
\begin{aligned}
& x_{i}(t)=x_{i}^{0}(t) e_{0}+x_{i}^{1}(t) e_{1}+x_{i}^{2}(t) e_{2}+x_{i}^{12}(t) e_{12}, \\
& f_{j}\left(x_{j}\right)=\frac{1}{25} e_{0} \sin ^{2} x_{j}^{1}+\frac{1}{30} e_{1} \tanh \left(x_{j}^{12}+x_{j}^{0}\right)+\frac{1}{35} e_{2} \sin x_{j}^{2}+\frac{1}{40} e_{12} \tanh ^{2} x_{j}^{0}, \\
& g_{j}\left(x_{j}\right)=\frac{1}{50} e_{0} \sin ^{2} x_{j}^{1}+\frac{1}{52} e_{1} \tanh \left(x_{j}^{12}+x_{j}^{0}\right)+\frac{1}{80} e_{2} \tanh ^{2} x_{j}^{2}+\frac{1}{55} e_{12} \sin x_{j}^{0}, \\
& h_{j}\left(x_{j}\right)=\frac{1}{100} e_{0} \sin ^{2} x_{j}^{0}+\frac{1}{50} e_{1} \sin \left(x_{j}^{1}+x_{j}^{0}\right)+\frac{1}{70} e_{2} \sin x_{j}^{2}+\frac{1}{60} e_{12} \sin ^{2} x_{j}^{12}, \\
& c_{1}(t)=(3.1-0.1 \sin \sqrt{5} t) e_{0}+(2.1+0.2 \sin t) e_{1}+(2-0.5 \cos \sqrt{3} t) e_{2}+(2+0.1 \sin t) e_{12} \text {, } \\
& c_{2}(t)=(3.1-0.1 \sin \sqrt{5} t) e_{0}+(2-0.5 \sin t) e_{1}+(2-0.1 \sin \sqrt{3} t) e_{2}+(2-0.4 \sin t) e_{12} \text {, } \\
& \left(\begin{array}{l}
I_{1}(t) \\
I_{2}(t)
\end{array}\right)=\left(\begin{array}{l}
2 e_{0} \sin t+e_{1} \cos \sqrt{3} t+0.3 e_{2} \sin \sqrt{5} t+2 e_{12} \sin t \\
\left(2 \sin t+\frac{2}{1+t^{2}}\right) e_{0}+0.5 e_{1} \cos \sqrt{3} t+0.2 e_{2} \sin \sqrt{2} t+2 e_{12} \cos t
\end{array}\right), \\
& \left(\begin{array}{ll}
a_{11}(t) & a_{12}(t) \\
a_{21}(t) & a_{22}(t)
\end{array}\right)=\left(\begin{array}{ll}
0.02 e_{0} \sin \sqrt{2} t+0.03 e_{1} \sin t & 0.05 e_{2} \sin \sqrt{2} t+0.05 e_{12} \cos \sqrt{3} t \\
0.02 e_{1} \sin \sqrt{2} t+0.08 e_{2} \sin t & 0.07 e_{0} \cos t+0.09 e_{12} \sin \sqrt{3} t
\end{array}\right), \\
& \left(\begin{array}{ll}
b_{11}(t) & b_{12}(t) \\
b_{21}(t) & b_{22}(t)
\end{array}\right)=\left(\begin{array}{ll}
0.01 e_{0} \sin t+0.06 e_{1} \cos \sqrt{5} t & 0.08 e_{2} \sin \sqrt{7} t+0.09 e_{12} \cos \sqrt{3} t \\
0.05 e_{1} \sin t+0.11 e_{2} \cos \sqrt{2} t & 0.01 e_{0} \cos \sqrt{6} t+0.03 e_{12} \sin \sqrt{5} t
\end{array}\right), \\
& \left(\begin{array}{ll}
\alpha_{11}(t) & \alpha_{12}(t) \\
\alpha_{21}(t) & \alpha_{22}(t)
\end{array}\right)=\left(\begin{array}{ll}
0.01 e_{0} \sin \sqrt{3} t+0.02 e_{1} \sin t & 0.02 e_{2} \cos \sqrt{2} t+0.05 e_{12} \sin \sqrt{3} t \\
0.02 e_{1} \sin t+0.03 e_{2} \sin \sqrt{2} t & 0.03 e_{0} \sin \sqrt{3} t+0.01 e_{12} \cos \sqrt{2} t
\end{array}\right), \\
& \left(\begin{array}{ll}
\beta_{11}(t) & \beta_{12}(t) \\
\beta_{21}(t) & \beta_{22}(t)
\end{array}\right)=\left(\begin{array}{ll}
0.03 e_{0} \sin t+0.02 e_{1} \sin \sqrt{3} t & 0.02 e_{2} \sin \sqrt{2} t+0.03 e_{12} \sin \sqrt{3} t \\
0.08 e_{1} \sin \sqrt{2} t+0.02 e_{2} \cos \sqrt{3} t & 0.01 e_{0} \sin \sqrt{3} t+0.04 e_{12} \sin \sqrt{7} t
\end{array}\right),
\end{aligned}
$$




$$
\begin{gathered}
\left(\begin{array}{ll}
S_{11}(t) & S_{12}(t) \\
S_{21}(t) & S_{22}(t)
\end{array}\right)=\left(\begin{array}{ll}
0.05 e_{0} \sin \sqrt{7} t+0.01 e_{1} \sin \sqrt{3} t & 0.07 e_{2} \sin \sqrt{7} t+0.02 e_{12} \sin \sqrt{3} t \\
0.03 e_{1} \sin \sqrt{3} t+0.02 e_{2} \sin \sqrt{2} t & 0.03 e_{0} \cos \sqrt{2} t+0.05 e_{12} \sin \sqrt{3} t
\end{array}\right) \\
\left(\begin{array}{ll}
T_{11}(t) & T_{12}(t) \\
T_{21}(t) & T_{22}(t)
\end{array}\right)=\left(\begin{array}{ll}
0.01 e_{0} \sin \sqrt{2} t+0.06 e_{1} \sin t & 0.05 e_{2} \sin \sqrt{3} t+0.05 e_{12} \cos \sqrt{2} t \\
0.02 e_{1} \sin t+0.03 e_{2} \sin \sqrt{2} t & 0.07 e_{0} \cos t+0.09 e_{12} \sin \sqrt{3} t
\end{array}\right), \\
v_{1}(t)=v_{2}(t)=\frac{1}{11} e_{0} \sin \sqrt{3} t+\frac{1}{12} e_{1} \cos \sqrt{2} t+\frac{1}{10} e_{2} \sin \sqrt{5} t+\frac{1}{15} e_{12} \sin t \\
q_{11}=0.5, q_{12}=0.4, q_{21}=0.4, q_{22}=0.3 .
\end{gathered}
$$

By a simple calculation, we have

$$
\begin{gathered}
K_{j}=\frac{1}{25}, L_{j}=\frac{1}{50}, M_{j}=\frac{1}{50}, \bar{c}_{1}^{\varnothing}=\bar{c}_{2}^{\varnothing}=3, \bar{c}_{1}^{c}=2.2, \bar{c}_{2}^{c}=2.5, q=0.3, \\
a_{11}^{+}=0.03, a_{12}^{+}=0.05, a_{21}^{+}=0.08, a_{22}^{+}=0.09, b_{11}^{+}=0.06, b_{12}^{+}=0.09, b_{21}^{+}=0.05, b_{22}^{+}=0.03, \\
\alpha_{11}^{+}=0.02, \alpha_{12}^{+}=0.05, \alpha_{21}^{+}=0.03, \alpha_{22}^{+}=0.03, \beta_{11}^{+}=0.03, \beta_{12}^{+}=0.03, \beta_{21}^{+}=0.08, \beta_{22}^{+}=0.04 .
\end{gathered}
$$

Besides, we can get

$$
\begin{aligned}
& \frac{1}{\bar{c}_{1}^{\varnothing}}\left(\bar{c}_{1}^{c}+\sum_{j=1}^{2} a_{1 j}^{+} K_{j}+\sum_{j=1}^{2} b_{1 j}^{+} L_{j}+\sum_{j=1}^{2} \alpha_{1 j}^{+} M_{j}+\sum_{j=1}^{2} \beta_{1 j}^{+} M_{j}\right) \approx 0.7364<1, \\
& \frac{1}{\bar{c}_{2}^{\varnothing}}\left(\bar{c}_{2}^{c}+\sum_{j=1}^{2} a_{2 j}^{+} K_{j}+\sum_{j=1}^{2} b_{2 j}^{+} L_{j}+\sum_{j=1}^{2} \alpha_{2 j}^{+} M_{j}+\sum_{j=1}^{2} \beta_{2 j}^{+} M_{j}\right) \approx 0.8373<1
\end{aligned}
$$

and $\delta \approx 0.8373<1$. Hence, conditions $\left(H_{1}\right)-\left(H_{3}\right)$ of are verified. Consequently, by Theorem 2, system (1) possesses a unique one pseudo almost periodic solution that is globally attractive (see Figures 1-4).

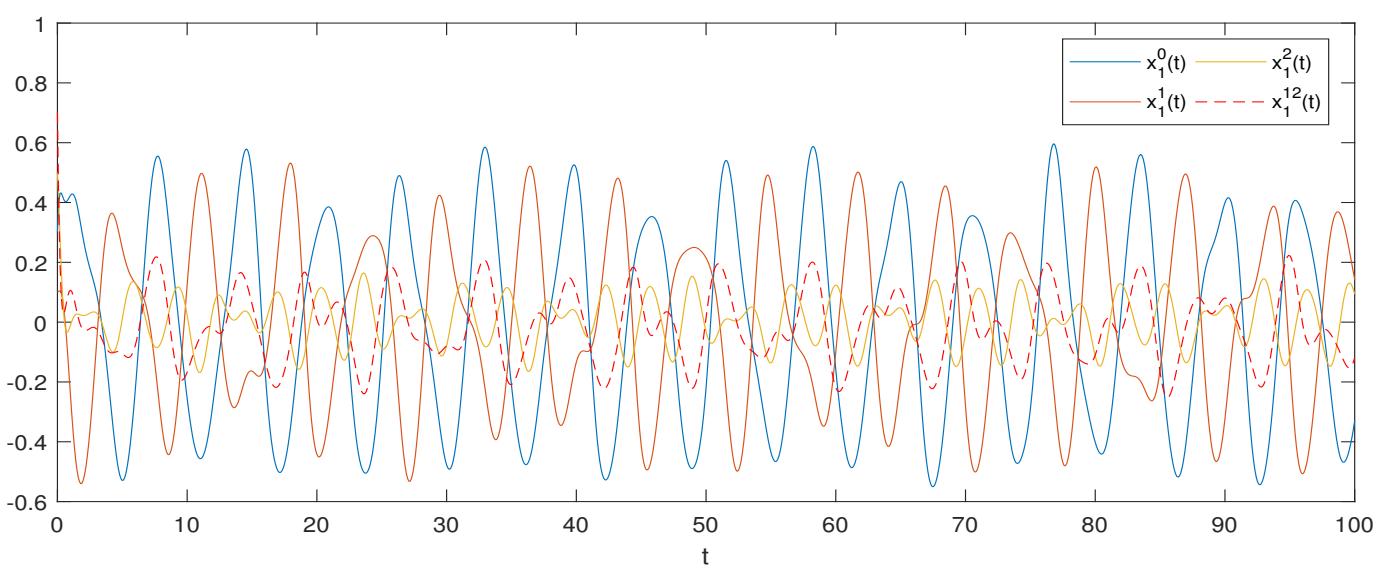

Figure 1. The states $x_{1}^{0}, x_{1}^{1}, x_{1}^{2}, x_{1}^{12}$ of (1) with different initial values. 


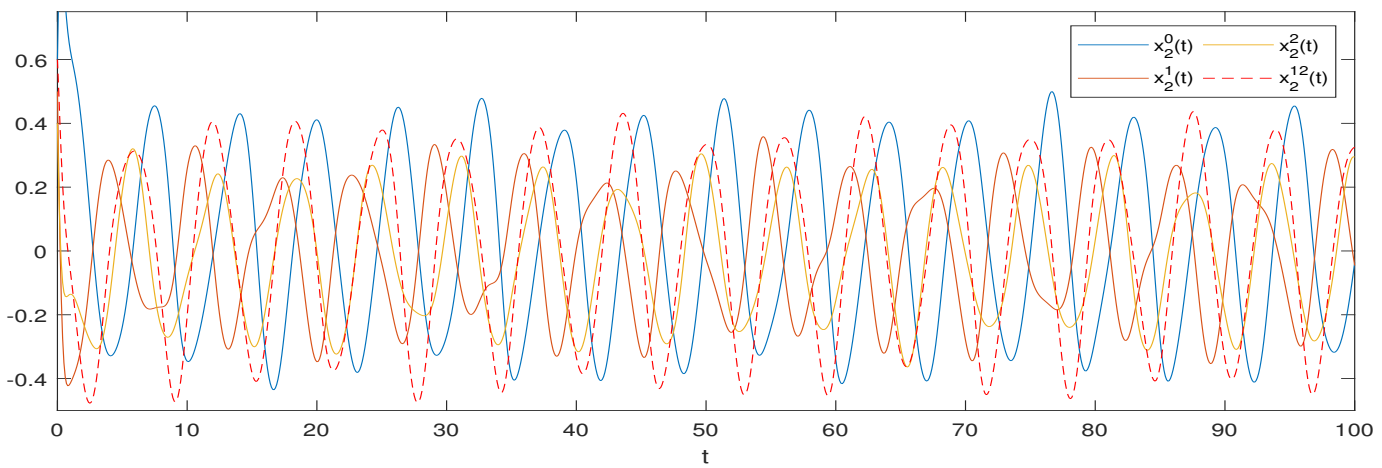

Figure 2. The states $x_{2}^{0}, x_{2}^{1}, x_{2}^{2}, x_{2}^{12}$ of (1) with different initial values.
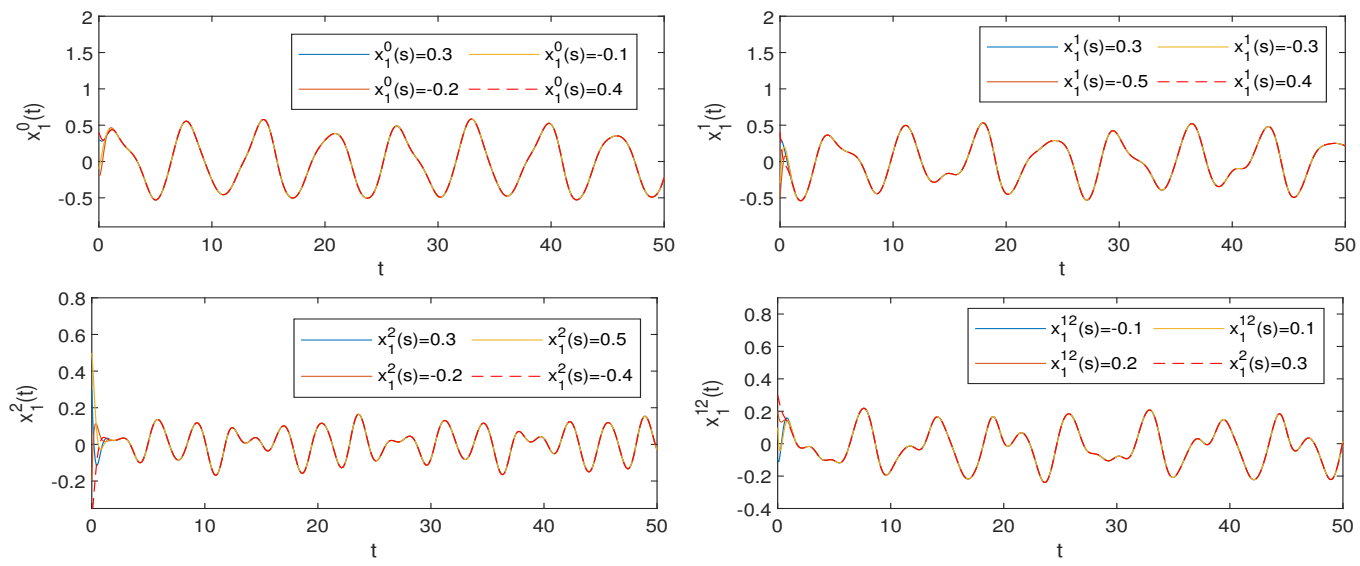

Figure 3. The stability of states $x_{1}^{0}, x_{1}^{1}, x_{1}^{2}, x_{1}^{12}$ of (1) with different initial values.
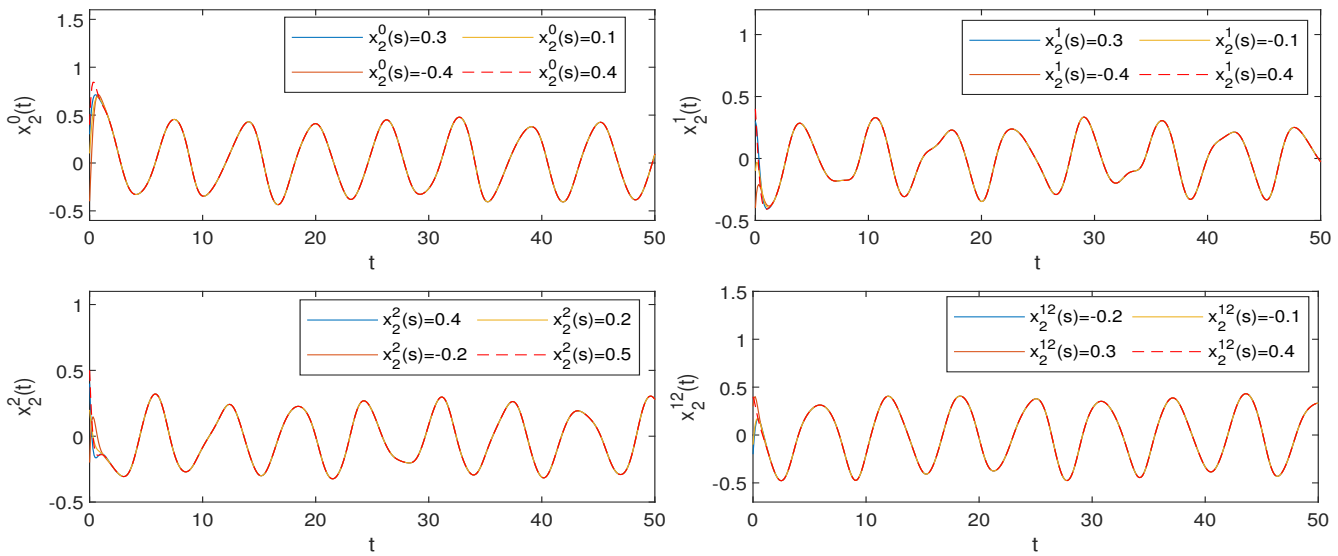

Figure 4 . The stability of states $x_{2}^{0}, x_{2}^{1}, x_{2}^{2}, x_{2}^{12}$ of (1) with different initial values.

Remark 1. No known results can be used to draw the conclusion of Example 1.

\section{Conclusions}

In this paper, we studied the existence and global attractivity of pseudo almost periodic solutions for a class of Clifford-valued fuzzy neural networks with proportional delays whose leakage coefficients were also Clifford numbers by the direct method. Our results are new. Our method can be used to study the existence and attractivity of pseudo almost periodic solutions for other types of Clifford-valued neural networks with proportional delays, and to study the existence and attractivity of almost automorphic solutions of Clifford-valued neural networks with proportional delays. 
Author Contributions: Conceptualization, B.L.; Investigation, W.L. and B.L.; Supervision, B.L.; Writing-original draft, W.L. and B.L.; Writing—review and editing, B.L. All authors have read and agreed to the published version of the manuscript.

Funding: This work is supported by the Applied Basic Research Foundation of Yunnan Province under Grant No. 2019FB003.

Institutional Review Board Statement: Not applicable.

Informed Consent Statement: Not applicable.

Data Availability Statement: Not applicable.

Conflicts of Interest: The authors declare no conflict of interest.

\section{References}

1. Buchholz, S. A Theory of Neural Computation with Clifford Algebras. Ph.D. Thesis, University of Kiel, Kiel, Germany, 2005.

2. Buchholz, S.; Sommer, G. On Clifford neurons and Clifford multi-layer perceptrons. Neural Netw. 2008, 21, 925-935. [CrossRef]

3. Liu, Y.; Xu, P.; Lu, J. Global stability of Clifford-valued recurrent neural networks with time delays. Nonlinear Dyn. 2016, 84, 767-777. [CrossRef]

4. Zhu, J.; Sun, J. Global exponential stability of Clifford-valued recurrent neural networks. Neurocomputing 2016, 173, 685-689. [CrossRef]

5. Li, Y.; Xiang, J. Global asymptotic almost periodic synchronization of Clifford-valued CNNs with discrete delays. Complexity 2019, 2019, 6982109. [CrossRef]

6. Huo, N.; Li, B.; Li, Y. Anti-periodic solutions for Clifford-valued high-order Hopfield neural networks with state-dependent and leakage delays. Int. J. Appl. Math. Comput. Sci. 2020, 30, 83-98.

7. Li, Y.; Huo, N.; Li, B. On $\mu$-pseudo almost periodic solutions for Clifford-valued neutral type neural networks with delays in the leakage term. IEEE Trans. Neural Netw. Learn. Syst. 2021, 32, 1365-1374. [CrossRef] [PubMed]

8. Li, Y.; Shen, S. Pseudo almost periodic synchronization of Clifford-valued fuzzy cellular neural networks with time-varying delays on time scales. Adv. Differ. Equ. 2020, 2020, 593. [CrossRef]

9. Li, Y.; Li, B. Pseudo compact almost automorphy of neutral type Clifford-valued neural networks with mixed delays. Discrete Contin. Dyn. Syst.-B 2021, in press. [CrossRef]

10. Aouiti, C.; Dridi, F.; Hui, Q.; Moulay, E. $(\mu, v)$-pseudo almost automorphic solutions of neutral type Clifford-valued high-Order Hopfield neural networks with D Operator. Neural Process. Lett. 2021, 53, 799-828. [CrossRef]

11. Aouiti, C.; Dridi, F. Weighted pseudo almost automorphic solutions for neutral type fuzzy cellular neural networks with mixed delays and D operator in Clifford algebra. Int. J. Syst. Sci. 2020, 51, 1759-1781. [CrossRef]

12. Liu, Y. Asymptotic behavior of functional differential equations with priportional time delays. Eur. J. Appl. Math. 1996, 7, 11-30. [CrossRef]

13. Huang, Z. Almost periodic solutions for fuzzy cellular neural networks with multi-proportional delays. Int. J. Mach. Learn. Cyber 2017, 8, 1323-1331. [CrossRef]

14. Liang, J.; Qian, H.; Liu, B. Pseudo almost periodic solutions for fuzzy cellular neural networks with multi-proportional delays. Neural Process. Lett. 2018, 48, 1201-1212. [CrossRef]

15. Huang, C.; Long, X.; Cao, J. Stability of antiperiodic recurrent neural networks with multiproportional delays. Math. Meth. Appl. Sci. 2020, 43, 6093-6102. [CrossRef]

16. Wang, W. Finite-time synchronization for a class of fuzzy cellular neural networks with time-varying coefficients and proportional delays. Fuzzy Sets Syst. 2018, 338, 40-49. [CrossRef]

17. Qin, J.; Li, Y. New results on exponential stability of competitive neural networks with multi-proportional delays. Asian J. Control 2020, 22, 750-760. [CrossRef]

18. Yang, T.; Yang, L.; Wu, C.; Chua, L. The global stability of fuzzy cellular neural network. IEEE Trans. Circ. Syst. I 1996, 43, 880-883. [CrossRef]

19. Shen, S.; Li, Y. $S^{p}$-almost periodic solutions of Clifford-valued fuzzy cellular neural networks with time-varying delays. Neural Process. Lett. 2020, 51, 1749-1769. [CrossRef]

20. Li, B.; Li, Y. Existence and global exponential stability of pseudo almost periodic solution for Clifford-valued neutral high-order Hopfield neural networks with leakage delays. IEEE Access 2019, 7, 150213-150225. [CrossRef]

21. Yu, Y. Exponential stability of pseudo almost periodic solution for cellular neural networks with multi-proportional delays. Neural Process. Lett. 2017, 45, 141-151. [CrossRef]

22. Meng, X.; Li, Y. Pseudo almost periodic solutions for quaternion-valued high-order Hopfield neural networks with time-varying delays and leakage delays on time scales. AIMS Math. 2021, 6, 10070-10091. [CrossRef]

23. Huang, C.; Liu, B.; Qian, C.; Cao, J. Stability on positive pseudo almost periodic solutions of HPDCNNs incorporating D operator. Math. Comput. Simul. 2021, 190, 1150-1163. [CrossRef] 
24. Arbi, A. Dynamics of BAM neural networks with mixed delays and leakage time-varying delays in the weighted pseudo-almost periodic on time-space scales. Math. Meth. Appl. Sci. 2018, 41, 1230-1255. [CrossRef]

25. Tang, Y. Pseudo almost periodic shunting inhibitory cellular neural networks with multi-proportional delays. Neural Process. Lett. 2018, 48, 167-177. [CrossRef]

26. Kong, F.; Fang, X. Pseudo almost periodic solutions of discrete-time neutral-type neural networks with delays. Appl. Intell. 2018, 48, 3332-3345. [CrossRef]

27. Li, Y.; Wang, X. Almost periodic solutions in distribution of Clifford-valued stochastic recurrent neural networks with time-varying delays. Chaos Solitons Fractals 2021, 153, 111536. [CrossRef]

28. Aouiti, C.; Gharbia, I.B. Piecewise pseudo almost-periodic solutions of impulsive fuzzy cellular neural networks with mixed delays. Neural Process. Lett. 2020, 51, 1201-1225. [CrossRef]

29. Tang, Y. Exponential stability of pseudo almost periodic solutions for fuzzy cellular neural networks with time-varying delays. Neural Process. Lett. 2019, 49, 851-861. [CrossRef]

30. Li, Y.; Huo, N. $(\mu, v)$-pseudo almost periodic solutions of Clifford-valued high-order HNNs with multiple discrete delays. Neurocomputing 2020, 414, 1-9. [CrossRef] 